\title{
Compact Homomorphisms on Algebras of Continuous Functions
}

\author{
Junzo WADA \\ Waseda University
}

\section{Introduction.}

The purpose of this note is to study compact and weakly compact homomorphisms between algebras of continuous functions. For a completely regular Hausdorff space $S$, we denote by $C(S)$ the algebra of all complex-valued continuous functions on $S$ endowed with its compact-open topology. M. Lindström and J. Llavana [4] gave characterizations of compact and weakly compact homomorphisms from $C(S)$ to $C(T)$, where $T$ and $S$ are completely regular Hausdorff spaces. Let $A$ and $B$ be closed subalgebras of $C(S)$ and $C(T)$ respectively. Here we study compact and weakly compact homomorphisms $\varphi$ from $A$ to $B$.

After some preliminaries in $\S 1$, we introduce in $\S 2$ closed subalgebras of some type which are called function algebras induced by uniform algebras. These subalgebras contain $C(S)$ and algebras of analytic functions. We discuss in $\S 2$ compactness and weak compactness of $\varphi$ in the case $A$ is a function algebra induced by a uniform algebra and $\varphi$ is a composition operator. We give conditions under $\varphi$ is compact or weakly compact and establish the relationship between compactness and weak compactness of $\varphi$.

\section{§1. Preliminaries.}

For a completely regular Hausdorff space $X$, we denote by $C(X)$ the algebra of all complex-valued continuous functions on $X$ endowed with its compact-open topology. Throughout this note we let $S$ and $T$ denote completely regular Hausdorff spaces.

Let $A$ and $B$ be subalgebras of $C(S)$ and $C(T)$ respectively. Then we easily have the following (cf. [6], [8]).

(a) Let $\varphi$ be a continuous linear operator from $A$ to $B$. Then there is a continuous mapping $\tau$ from $T$ to the dual space $A^{\prime}$ of $A$ with respect to the $w^{*}$-topology $\sigma\left(A^{\prime}, A\right)$ such that

$$
[\varphi(f)](y)=\tau(y)(f), \quad f \in A \text { and } y \in T .
$$

(b) Let $\varphi$ be a continuous homomorphism from $A$ to $B$. Then there is a continuous

Received July 5, 1994 
mapping $\tau$ from $T$ to $A^{\prime}$ with respect to the topology $\sigma\left(A^{\prime}, A\right)$ such that (*) is satisfied and $\tau(y)$ is a continuous homomorphism from $A$ to the complex field $C$ for any $y \in T$.

Let $X$ be a completely regular Hausdorff space. We say that $A$ is a function algebra on $X$ if it is a closed subalgebra of $C(X)$ separating points in $X$ and containing constant functions.

Now let $A$ and $B$ be function algebras on $S$ and $T$ respectively and let $\varphi$ be a continuous homomorphism from $A$ to $B$. The homomorphism $\varphi$ is called a composition operator if there is a continuous mapping $\theta$ from $T$ to $S$ such that

$$
[\varphi(f)](y)=f(\theta(y)), \quad f \in A \text { and } y \in T .
$$

Let $A$ be a function algebra on $S$. We consider the following conditions on $A$ :

(1) For any non-trivial continuous homomorphism $\psi$ from $A$ to $C$, there is an $\alpha \in S$ such that $\psi(f)=f(\alpha)$ for any $f \in A$.

(2) For any $x_{0} \in S$ and any open neighborhood $V$ of $x_{0}$, we can find a finite number of functions $f_{1}, f_{2}, \cdots, f_{n}$ in $A$ and $\delta>0$ such that $f_{i}\left(x_{0}\right)=0(i=1,2, \cdots, n)$ and $\bigcap_{i=1}^{n}\left\{x \in S:\left|f_{i}(x)\right|<\delta\right\} \subset V$.

The conditions (1) and (2) guarantee that any continuous homomorphism $\varphi$ from $A$ to $B$ with $\varphi(1)=1$ is a composition operator. We easily see the following.

LEMMA 1.1. Let $A$ and $B$ be function algerbas on completely regular Hausdorff spaces $S$ and $T$ respectively. Suppose that $A$ satisfies conditions (1) and (2). If $\varphi$ is a continuous homomorphism from $A$ to $B$ with $\varphi(1)=1$, then $\varphi$ is a composition operator.

Proof. For any $y \in T, \tau(y)$ in (*) is a non-trivial continuous homomorphism from $A$ to $C$ by (b). Since $A$ satisfies condition (1), there is an $\alpha \in S$ such that $\tau(y)(f)=f(\alpha)$ for any $f \in A$. This $\alpha$ is uniquely determined since $A$ separates points of $S$. If we put $\theta(y)=\alpha, \theta$ is a mapping from $T$ to $S$ and $[\varphi(f)](y)=f(\theta(y))$ for $f \in A$ and $y \in T$. It remains only to show that $\theta$ is continuous. If $y_{\lambda} \rightarrow y$ in $T$, then $\tau\left(y_{\lambda}\right) \rightarrow \tau(y)$ in $\sigma\left(A^{\prime}, A\right)$. So $f\left(\theta\left(y_{\lambda}\right)\right)=\tau\left(y_{\lambda}\right)(f) \rightarrow \tau(y)(f)=f(\theta(y))$ for $f \in A$. From condition (2), for any open neighborhood $V$ of $\theta(y)$, there are $f_{1}, f_{2}, \cdots, f_{n} \in A$ and $\delta>0$ such that $f_{i}(\theta(y))=0$ $(i=1,2, \cdots, n)$ and $\bigcap_{i=1}^{n}\left\{x \in S:\left|f_{i}(x)\right|<\delta\right\} \subset V$. Since $f_{i}(\theta(y))=0,\left|f_{i}\left(\theta\left(y_{\lambda}\right)\right)\right|<\delta$ for any $\lambda \geq \lambda_{0}$ for some $\lambda_{0}(i=1,2, \cdots, n)$. Hence $\theta\left(y_{\lambda}\right) \in V$ for $\lambda \geq \lambda_{0}$ and $\theta$ is continuous.

EXAMPLeS. (i) Let $S$ be a completely regular Hausdorff space. Then $A=C(S)$ satisfies condition (1) (cf. [3]). $A$ evidently satisfies condition (2).

(ii) Let $G$ be an open subset in $C$ and let $A=H(G)$ be the algebra of all analytic functions on $G$. Then $H(G)$ satisfies conditions (1) and (2).

(iii) Let $G$ be an open polydisc in $C^{2}$ and let $H(G)$ be the algebra of all analytic functions on $G$. Then $H(G)$ has condition (1). The algebra $H(G)$ also satisfies condition (2).

(iv) Let $X=D \times J$, where $D=\{z \in C:|z|<1\}$ and $J=(0,1)$. We put $A=\{f \in C(X)$ : $f$ can be approximated uniformly by polynomials in $z$ and $t$ on any compact subset of $X$ \}. Let $\varphi$ be a non-trivial continuous homomorphism from $A$ to $C$. If $\varphi(z)=\alpha$ and 
$\varphi(t)=\beta$, then $\beta$ is a real number and $(\alpha, \beta) \in X$, where $z, t$ are the coordinate functions. This shows that $A$ satisfies condition (1). One sees that $A$ satisfies condition (2).

Let $S$ be a completely regular Hausdorff space. We say that $S$ is a $k_{R}$-space if a complex-valued function $f$ on $S$ is continuous whenever $f \mid F$ is continuous on $F$ for any compact subset $F$ of $S$. The space $C(S)$ is complete if and only if $S$ is a $k_{R}$-space (cf. [9]). A subset $H$ of $C(S)$ is called equicontinuous if for any $x \in S$ and any $\varepsilon>0$, there is an open neighborhood $V$ of $x$ such that $|f(y)-f(x)|<\varepsilon$ for any $f \in H$ and any $y \in V$.

In order to give a characterization of compact homomorphisms from $A$ to $B$ we will need the following compactness criteria of Arzela-Ascoli type (cf. [4]).

LEMMA 1.2. Let $S$ be a completely regular Hausdorff $k_{R^{-}}$-space. Then a subset $H$ of $C(S)$ is relatively compact if and only if (i) $H$ is equicontinuous on $S$ and (ii) $\{f(x): f \in H\}$ is bounded in $C$ for any $x \in S$.

For given two locally convex spaces $E$ and $F$, we call a continuous linear operator $\varphi$ from $E$ to $F$ is compact (resp. weakly compact) if it maps bounded subsets of $E$ to relatively compact (resp. relatively weakly compact) subsets of $F$.

Let $A$ and $B$ be function algebras on $S$ and $T$ respectively, and let $\varphi$ be a composition operator from $A$ to $B$, that is, $\varphi$ is of the form $\varphi(f)=f \circ \theta$, where $\theta$ is a continuous mapping from $T$ to $S$. It is not hard to see the following.

LEMMA 1.3. Let $S$ and $T$ be completely regular Hausdorff spaces and assume that $T$ is a $k_{R}$-space. Let $A$ and $B$ be function algebras on $S$ and $T$ respectively. Then $a$ composition operator $\varphi$ from $A$ to $B$ is compact if and only if for any $y \in T$ any net $y_{\alpha} \rightarrow y$ in $T$ implies that $\sup _{f \in F}\left|f\left(\theta\left(y_{\alpha}\right)\right)-f(\theta(y))\right| \rightarrow 0$ for any bounded set $F$ in $A$.

Proof. $M(\subset B)$ is equicontinuous on $T$ if and only if for any $y$ in $T$, any net $y_{\alpha} \rightarrow y$ in $T$ implies that $\sup _{g \in M}\left|g\left(y_{\alpha}\right)-g(y)\right| \rightarrow 0$. Hence the lemma is clear by Lemma 1.2 since $B$ is closed in $C(T)$.

\section{§2. Compact and weakly compact composition operators.}

Let $A_{0}$ be a uniform algebra on the maximal ideal space $M_{A_{0}}$ of $A_{0}$. Let $\mathfrak{P}_{0}$ be the family of all Gleason parts for $A_{0}$ and let $S$ be the union of members of a subfamily $\mathfrak{P}$ of $\mathfrak{P}_{0}$, that is, $S=\bigcup_{P \in \mathfrak{P}} P$. Here $S$ is a completely regular Hausdorff space as a subspace of $M_{A_{0}}$. We set $A=\{f \in C(S): f$ can be approximated uniformly on $F$ by functions in $A_{0} \mid S$ for any compact subset $F$ of $\left.S\right\}$. Such an $A$ is called the function algebra on $S$ induced by $A_{0}$. It is the smallest closed subalgebra in $C(S)$ containing $A_{0} \mid S$. We say that $P$ is a non-trivial part for $A$ if it is a non-trivial Gleason part for $A_{0}$ which is in $\mathfrak{P}$, and $P$ is a trivial part for $A$ if it is a trivial Gleason part for $A_{0}$ and $P \in \mathfrak{P}$.

EXAMPLES. (i) Let $S$ be a completely regular Hausdorff space and let $\beta S$ be the Čech compactification of $S$. Then $C(S)$ is the function algebra on $S$ induced by the 
uniform algebra $C(\beta S)$. Any part for $C(S)$ is trivial.

(ii) Let $D=\{z \in C:|z|<1\}$ and let $A_{0}$ be the disc algebra on $\bar{D}$. Then $A=H(D)$ is the function algebra on $D$ induced by $A_{0}$ and $D$ is the unique part for $A$.

(iii) Let $G=\left\{z=\left(z_{1}, z_{2}\right) \in C^{2}:\left|z_{1}\right|<1,\left|z_{2}\right|<1\right\}$ and let $A_{0}$ be the polydisc algebra on $\bar{G}$. Then $A=H(G)$ is the function algebra on $G$ induced by $A_{0}$ and $G$ is the unique part for $A$.

(iv) Let $X=D \times J$, where $D=\{z \in C:|z|<1\}$ and $J=(0,1)$. Let $A_{0}$ be the cylinder algebra, that is, $A_{0}=\{f \in C(\bar{D} \times[0,1]): f$ can be approximated uniformly by polynomials in $z$ and $t$ on $\bar{D} \times[0,1]\}$. Then $A$ on $D \times J$ of Example (iv) in $\S 1$ is the function algebra on $X$ induced by $A_{0}$ and any part for $A$ is of the form $D \times\{a\}$ for $a \in J$.

Let $A$ be the function algebra on $S$ induced by a uniform algebra $A_{0}$ and let $B$ be a function algebra on $T$. We consider a continuous homomorphism $\varphi$ from $A$ to $B$. Here we confine our attention to the case $\varphi$ is a composition operator. We are now in a position to discuss compactness of $\varphi$. First we have the following.

THEOREM 2.1. Let $A_{0}$ be a uniform algebra on $M_{A_{0}}$ and let $A$ be the function algebra on $S$ induced by $A_{0}$. Let $B$ be a function algebra on a completely regular Hausdorff $k_{R^{-}}$space $T$. Let $\varphi$ be a compact composition operator from $A$ to $B$. Then for any $y \in T$, there is an open neighborhood $U$ of $y$ such that $\theta(U)$ is contained in a part for $A$.

Proof. Suppose that there is a $y_{0} \in T$ such that $\theta(U)$ is not contained in the part $P$ for $A$ containing $\theta\left(y_{0}\right)$ for any open neighborhood $U$ of $y_{0}$. Let now $I$ be any fixed open neighborhood base at $y_{0}$ in $T$. Then the order relation $U \leq V$ if and only if $U \subset V$ directs $I$. If we take $y_{U} \in U$ such that $\theta\left(y_{U}\right) \notin P$ for any $U \in I$, then $\theta\left(y_{U}\right)$ is in a different part $P_{1}$ for $A$ from $P$. Hence $\sup \left\{\left|f\left(\theta\left(y_{U}\right)\right)-f\left(\theta\left(y_{0}\right)\right)\right|: f \in A_{0},\|f\| \leq 1\right\}=2$, and so for any $U \in I$, there is an $f_{U} \in A_{0},\left\|f_{U}\right\| \leq 1$ such that $1 \leq\left|f_{U}\left(\theta\left(y_{U}\right)\right)-f_{U}\left(\theta\left(y_{0}\right)\right)\right|$. Since $A_{0} \mid S \subset A, F=\left\{f_{U} \mid S: U \in I\right\}$ is a bounded set in $A$. From this,

$$
1 \leq\left|f_{U}\left(\theta\left(y_{U}\right)\right)-f_{U}\left(\theta\left(y_{0}\right)\right)\right| \leq \sup _{g \in F}\left|g\left(\theta\left(y_{U}\right)\right)-g\left(\theta\left(y_{0}\right)\right)\right|
$$

for any $U \in I$. Since $\varphi$ is compact and $y_{U} \rightarrow y_{0}$, this is a contradiction from Lemma 1.3.

REMARK. In Theorem 2.1 , if $T$ is connected, there is a uniquely determined part $P$ for $A$ which is independent of $y$ in $T$.

Next we will give the converse of Theorem 2.1 under a suitable assumption. We begin with explanation of the condition that $A$ has some analytic property. Let $P$ be a non-trivial part for the function algebra $A$ induced by a uniform algebra. We consider the following condition:

( $\alpha$ ) For any $x \in P$, there are an open neighborhood $V$ of $x$ in $P$ and a homeomorphism $\rho$ from the open unit polydisc $D^{n}$ (disc if $n=1, n$ depends on $x$ ) onto $V$ such that $f \circ \rho$ is analytic on $D^{n}$ for all $f \in A$. 
If any non-trivial part $P$ for $A$ satisfies this condition, then we say $A$ has $(\alpha)$. This property $(\alpha)$ can be found in [6].

Remark. All of function algebras $C(S), H(D), H(G)$ and $A$ on $D \times J$ of Examples in $\S 2$ have $(\alpha)$.

Here we wish to show the converse of Theorem 2.1 .

THEOREM 2.2. Suppose that the function algebra $A$ on $S$ induced by a uniform algebra $A_{0}$ has $(\alpha)$ and $S$ is a locally compact Hausdorff space. Let $\varphi$ be a continuous composition operator from $A$ to a function algebra $B$ on a completely regular Hausdorff $k_{R^{-}}$space $T$. If for any $y$ in $T$ there is an open neighborhood $U$ of $y$ such that $\theta(U)$ is contained in a part for $A$, then $\varphi$ is compact.

ProOF. From Lemma 1.3, in order to prove the theorem, it suffices to show that if $y_{\alpha} \rightarrow y$ in $T$, then $\sup _{f \in F}\left|f\left(\theta\left(y_{\alpha}\right)\right)-f(\theta(y))\right| \rightarrow 0$ for any bounded set $F$ in $A$. By the hypothesis, for any $y \in T$, there is an open neighborhood $U$ of $y$ such that $\theta(U)$ is contained in a part $P$ for $A$. Let $y_{\alpha} \rightarrow y$ in $T$ and let $F$ be a bounded set in $A$. If $\{\theta(y)\}$ is a trivial part for $A$, there is an open neighborhood $U$ of $y$ such that $\theta(U)=\{\theta(y)\}$. It is simple to check that $\sup _{f \in F}\left|f\left(\theta\left(y_{\alpha}\right)\right)-f(\theta(y))\right| \rightarrow 0$ as $y_{\alpha} \rightarrow y$. Next, let the part $P$ containing $\theta(y)$ be non-trivial. From $(\alpha)$, there is a homeomorphism $\rho$ from $D^{n}$ onto an open neighborhood $V$ of $\theta(y)$ in $P$ such that $f \circ \rho$ is analytic on $D^{n}$ for any $f \in A$. Since $S$ is locally compact, there is an open neighborhood $W$ of $\rho^{-1}(\theta(y))$ in $D^{n}$ such that $\rho(W)$ is an open neighborhood of $\theta(y)$ in $P$ and the closure $\overline{\rho(W)}$ of $\rho(W)$ in $S$ is compact. Since $F$ is a bounded set in $A$, $\sup _{f \in F}\|f\|_{\overline{\rho(W)}}<\infty$. Since $y_{\alpha} \rightarrow y, \theta\left(y_{\alpha}\right) \rightarrow \theta(y)$ in $S$. It implies that for some $\alpha_{0} \theta\left(y_{\alpha}\right) \in \rho(W)$ for every $\alpha \geq \alpha_{0}$ since $\theta(U) \subset P$. Here for any $\varepsilon>0$ there is an open neighborhood $W_{1}\left(W_{1} \subset W\right)$ of $\rho^{-1}(\theta(y))$ in $D^{n}$ such that for any $z \in W_{1}$ and any $f \in F$

$$
\left|f(\rho(z))-f\left(\rho\left(\rho^{-1}(\theta(y))\right)\right)\right|<\varepsilon,
$$

since $f \circ \rho$ is analytic on $W$ for any $f \in F$ and $\sup _{f \in F}\|f\|_{\overline{\rho(W)}}<\infty$. Now since $\theta\left(y_{\alpha}\right) \in \rho\left(W_{1}\right)$ for $\alpha \geq$ some $\alpha_{1}, \theta\left(y_{\alpha}\right)=\rho\left(z_{\alpha}\right)$ for a $z_{\alpha} \in W_{1}\left(\alpha \geq \alpha_{1}\right)$. Hence

$$
\sup _{f \in F}\left|f\left(\theta\left(y_{\alpha}\right)\right)-f(\theta(y))\right|=\sup _{f \in F}\left|f\left(\rho\left(z_{\alpha}\right)\right)-f\left(\rho\left(\rho^{-1}(\theta(y))\right)\right)\right|<\varepsilon
$$

for $\alpha \geq \alpha_{1}$. The proof is completed.

Remarks. (1) When $A=C(S)$, the hypotheses in Theorem 2.2 that $S$ is locally compact and $A$ has $(\alpha)$ are unnecessary since any part for $A$ is trivial. Hence if $A=C(S)$, we have the following by Theorems 2.1 and 2.2: Assume that $S$ and $T$ are completely regular Hausdorff spaces and $T$ is a $k_{R}$-space. A continuous homomorphism $\varphi$ from $C(S)$ to $C(T)$ is compact if and only if $\theta$ is locally constant. This was given in [4, Proposition 3].

(2) The identity mapping $\varphi$ from $H(D)$ to itself is compact by Theorem 2.2 . This 
also can be seen in [5]. On the other hand, if $A$ is the function algebra on $S=D \cup\{1\}$ induced by the disc algebra on $\bar{D}$, then the identity mapping from $A$ to itself is not compact by Theorem 2.1.

Our next aim is to find out conditions for a weakly compact composition operator to be compact. We begin with the following theorem. It is proved by using a way similar to the argument in [7, Theorem 1].

THEOREM 2.3. Let $A$ be the function algebra on $S$ induced by a uniform algebra $A_{0}$ and let $B$ be a function algebra on a locally compact Hausdorff space T. Let $\varphi$ be a weakly compact composition operator from $A$ to $B$. Then for any $y \in T$, there is an open neighborhood $U$ of $y$ such that $\theta(U)$ is contained in a part for $A$.

Proof. For any $y_{0} \in T$, let $P_{0}$ be the part for $A$ containing $\theta\left(y_{0}\right)$. Put $W=\left\{z \in T: \theta(z) \in P_{0}\right\}$. In order to prove the theorem, it suffices to show that $W$ is a neighborhood of $y_{0}$. Assume the contrary. Then $W$ does not contain any open neighborhood of $y_{0}$. Since $T$ is locally compact, we can choose an open neighborhood $V_{1}$ of $y_{0}$ such that $\bar{V}_{1}$ is compact. Put $\varepsilon_{n}=1 /(n+2)^{2}$ for any $n$. Now if we take a $y_{1}$ in $V_{1} \backslash W$, then $\theta\left(y_{1}\right)$ is in a different part for $A$ from $P_{0}$. Hence there is an $f_{1} \in A_{0},\left\|f_{1}\right\| \leq 1$ such that $\left[\theta\left(y_{1}\right)\right]\left(f_{1}\right)=0,\left[\theta\left(y_{0}\right)\right]\left(f_{1}\right)>1-\varepsilon_{1}$. Put $V_{2}=\left\{z \in V_{1}:\left|[\theta(z)]\left(f_{1}\right)\right|>1-\varepsilon_{1}\right\}$. Then $V_{2}$ is an open neighborhood of $y_{0}$. Take $y_{2} \in V_{2} \backslash W$. Then $\theta\left(y_{2}\right)$ is in a different part for $A$ from $P_{0}$. So there is a $g_{2} \in A_{0},\left\|g_{2}\right\| \leq 1$ such that $\left[\theta\left(y_{2}\right)\right]\left(g_{2}\right)=0$, $\left[\theta\left(y_{0}\right)\right]\left(g_{2}\right)>1-\varepsilon_{2}$. Set $f_{2}=f_{1} g_{2}$. Then $\left[\theta\left(y_{1}\right)\right]\left(f_{2}\right)=\left[\theta\left(y_{2}\right)\right]\left(f_{2}\right)=0, \quad\left[\theta\left(y_{0}\right)\right]\left(f_{2}\right)>$ $\left(1-\varepsilon_{1}\right)\left(1-\varepsilon_{2}\right)$ and $\left|\left[\theta\left(y_{2}\right)\right]\left(f_{1}\right)\right|>1-\varepsilon_{1}$. Continuing this process, we obtain a sequence $\left\{y_{n}\right\}$ in $V_{1}$ and a sequence $\left\{f_{n}\right\}$ in $A_{0}$ such that $\left\|f_{n}\right\| \leq 1$ for any $n$, and

$$
\begin{gathered}
{\left[\theta\left(y_{m}\right)\right]\left(f_{n}\right)=0 \quad(1 \leq m \leq n)} \\
{\left[\theta\left(y_{0}\right)\right]\left(f_{n}\right)>\left(1-\varepsilon_{1}\right)\left(1-\varepsilon_{2}\right) \cdots\left(1-\varepsilon_{n}\right)} \\
\left|\left[\theta\left(y_{n}\right)\right]\left(f_{m}\right)\right|>\left(1-\varepsilon_{1}\right) \cdots\left(1-\varepsilon_{m}\right) \quad(1 \leq m<n) .
\end{gathered}
$$

Since $\left\{y_{n}\right\}$ is contained in the compact set $\bar{V}_{1}$, there is a cluster point $z_{0}$ of $\left\{y_{n}\right\}$. Let $\left\{y_{\alpha}\right\}$ be a net converging to $z_{0}$ which consists of the members of $\left\{y_{n}\right\}$. By weak compactness of $\varphi$, the weak closure $F$ of $\left\{\varphi\left(f_{n} \mid S\right)\right\}$ in $B$ is a weakly compact subset in $B$, since $\left\{f_{n} \mid S\right\}$ is a bounded set in $A$. For any $g \in F, g\left(y_{\alpha}\right) \rightarrow g\left(z_{0}\right)$. Now put $\hat{y}_{\alpha}(g)=g\left(y_{\alpha}\right)$ and $\hat{z}_{0}(g)=g\left(z_{0}\right)$ for any $g \in F$. Then $\hat{y}_{\alpha}$ and $\hat{z}_{0}$ are continuous functions on $F$ with respect to the weak topology $\sigma\left(B, B^{\prime}\right)$. Since $\hat{y}_{\alpha}(f) \rightarrow \hat{z}_{0}(f)$ for any $f \in F$ and $F$ is a compact Hausdorff space with respect to $\sigma\left(B, B^{\prime}\right), \hat{y}_{\alpha}$ converges quasi-uniformly to $\hat{z}_{0}$ on $F$ (cf. [1, p. 268]). Hence for $0<\varepsilon<1 / 4$, and for any $\alpha_{0}$, there are $\alpha_{1}, \alpha_{2}, \cdots, \alpha_{m} \geq \alpha_{0}$ such that

$$
\min _{1 \leq i \leq m}\left|\hat{y}_{\alpha_{i}}(g)-\hat{z}_{0}(g)\right|<\varepsilon
$$

for any $g \in F$. In particular the inequality 


$$
\min _{1 \leq i \leq m}\left|\varphi\left(f_{n} \mid S\right)\left(y_{\alpha_{i}}\right)-\varphi\left(f_{n} \mid S\right)\left(z_{0}\right)\right|<\varepsilon<1 / 4
$$

holds for any $n$. Put $N=\max \left\{n_{1}, n_{2}, \cdots, n_{m}\right\}$, where $n_{i}$ is a positive integer such that $y_{\alpha_{i}}=y_{n_{i}}$. Then we have by (1)

$$
\varphi\left(f_{N} \mid S\right)\left(y_{n_{i}}\right)=f_{N}\left(\theta\left(y_{n_{i}}\right)\right)=\left[\theta\left(y_{n_{i}}\right)\right]\left(f_{N}\right)=0 \quad(i=1,2, \cdots, m) .
$$

Take an $M>N$ such that

$$
\left|f_{N}\left(\theta\left(y_{M}\right)\right)-f_{N}\left(\theta\left(z_{0}\right)\right)\right|<1 / 6
$$

Hence, from (1) and (3)

$$
\begin{aligned}
\left|f_{N}\left(\theta\left(z_{0}\right)\right)\right| & >\left|f_{N}\left(\theta\left(y_{M}\right)\right)\right|-1 / 6=\left|\left[\theta\left(y_{M}\right)\right]\left(f_{N}\right)\right|-1 / 6 \\
& >\left(1-\varepsilon_{1}\right)\left(1-\varepsilon_{2}\right) \cdots\left(1-\varepsilon_{N}\right)-1 / 6>1 / 3 .
\end{aligned}
$$

It follows that,

$$
\left|\varphi\left(f_{N} \mid S\right)\left(y_{n_{i}}\right)-\varphi\left(f_{N} \mid S\right)\left(z_{0}\right)\right|=\left|\varphi\left(f_{N} \mid S\right)\left(z_{0}\right)\right|=\left|f_{N}\left(\theta\left(z_{0}\right)\right)\right|>1 / 3
$$

holds for every $1 \leq i \leq m$. This contradicts (2) and the proof is completed.

Thus, by Theorems 2.2 and 2.3 , we can give conditions under any weakly compact composition operator from $A$ to $B$ is compact.

THEOREM 2.4. Let $A$ be the function algebra on $S$ induced by a uniform algebra $A_{0}$. Let $B$ be a function algebra on $T$. Assume that $S$ and $T$ are both locally compact Hausdorff spaces and $A$ satisfies the condition $(\alpha)$. Then any weakly compact composition operator from $A$ to $B$ is compact.

Finally we remark that if $A$ is even a closed subalgebra in $C(S)$ containing $A_{0} \mid S$ in place of a function algebra on $S$ induced by $A_{0}$, all of theorems in $\S 2$ hold true by defining that a part for $A$ is a member of $\mathfrak{P}$ as before.

\section{References}

[ 1 ] N. Dunford and J. T. Schwartz, Linear Operators, Part 1: General Theory, Interscience (1966).

[2] T. Gamelin, Uniform Algebras, Printice Hall (1969).

[3] T. Husain, Multiplicative Functionals on Topological Algebras, Pitman (1983).

[4] M. LindSTRÖm and J. LlavANA, Compact and weakly compact homomorphisms between algebras of continuous functions, J. Math. Anal. Appl. 166 (1992), 325-330.

[ 5] D. H. LuECKING and L. A. RuBel, Complex Analysis, A functional analysis approach, Springer (1984).

[6] S. OHNo and J. WADA, Compact homomorphisms on function algebras, Tokyo J. Math. 4 (1981), 105-112.

[7] H. TAKAGI and J. WADA, Weakly compact weighted composition operators on certain subspaces of $C(X, E)$, Proc. Japan Acad. 67 (1991), 304-307.

[ 8 ] J. WADA, Weakly compact linear operators on function spaces, Osaka Math. J. 13 (1961), $169-183$. 
[ 9 ] S. WARNER, The topology of compact convergence on continuous function spaces, Duke Math. J. 25 (1958), 265-282.

Present Address:

Department of Mathematics, School of Education, Waseda University, NishiwaSeda, ShinJUKU-KU, TOKYO, 169-50 JAPAN. 